\title{
Validation of CFD Model for Simulation of Spontaneous Ignition in Bio-mass Fuel Storage
}

\author{
ZHENGHUA YAN ${ }^{1}$, PER BLOMQVIST ${ }^{2}$, ULF GÖRANSSON ${ }^{3}$, \\ GÖRAN HOLMSTEDT ${ }^{3}$, LARS WADSÖ ${ }^{4}$, and PATRICK VAN HEES ${ }^{2}$ \\ ${ }^{1}$ Department of Building Science \\ Lund University, Sweden \\ ${ }^{2}$ Swedish National Testing and Research Institute \\ Borås, Sweden \\ ${ }^{3}$ Department of Fire Safety Engineering \\ Lund University, Sweden \\ ${ }^{4}$ Department of Building Material \\ Lund University, Sweden
}

\begin{abstract}
Both numerical simulations and experimental measurements of small scale spontaneous ignition with different biomass fuels have been performed. In the experiments, temperature history was monitored at five different locations inside the fuel bed. The measured temperature history was used for validation of comprehensive threedimensional computer simulations which were carried out using a parallel finite volume CFD code SMAFS (Smoke Movement and Flame Spread) developed by the first author.

The computation was based on numerical solution of a set of governing equations including the continuity equation, extended Darcy momentum equations, energy conservation equations for both gas and solid phases, and mass conservation equations for different chemical species. Consideration was given to a series of essential physical and chemical processes, including convection and diffusion in porous media, evaporation, condensation and heat generation which is mainly due to chemical oxidation. With reliable material properties input data provided by separate measurements, it simulated the temporal state evolution inside the biomass fuel storage. Numerical results were compared with experimental measurements, showing excellent agreement.
\end{abstract}

KEYWORDS: CFD simulation, experiment, spontaneous ignition

\section{NOMENCLATURE}

$\begin{array}{llll}A & \text { area }\left(\mathrm{m}^{2}\right) & t & \text { time }(\mathrm{s}) \\ a_{g i} & \text { gravity vector }\left(\mathrm{m} / \mathrm{s}^{2}\right) & u_{i}, u_{j} & \text { velocity vector }(\mathrm{m} / \mathrm{s}) \\ c_{F} & \text { form-drag constant } & W & \text { moisture content } \\ c_{p} & \text { specific heat capacity }(\mathrm{J} / \mathrm{kg} / \mathrm{K}) & Y_{i} & \text { mass fraction of species } i \\ D_{p} & \text { particle equivalent diameter }(\mathrm{m}) & \text { Greek } & \\ H & \text { enthalpy }(\mathrm{J} / \mathrm{kg}) & \mu & \text { dynamic viscosity }(\mathrm{kg} / \mathrm{m} / \mathrm{s}) \\ h & \text { height }(\mathrm{m}) & \rho & \text { density }\left(\mathrm{kg} / \mathrm{m}^{3}\right) \\ k & \text { permeability }\left(\mathrm{m}^{2}\right) & \varphi & \text { porosity } \\ P r & \text { Prandtl number } & \text { subscripts } & \\ p & \text { pressure }\left(\mathrm{N} / \mathrm{m}^{2}\right) & i & \text { co-ordinate or species } \\ r_{w} & \text { condensation rate }\left(\mathrm{kg} / \mathrm{m}^{3} / \mathrm{s}\right) & g & \text { gas } \\ S c & \text { Schmidt number } & \mathrm{s} & \text { solid }\end{array}$

FIRE SAFETY SCIENCE-PROCEEDINGS OF THE EIGHTH INTERNATIONAL SYMPOSIUM, pp. 151-162 


\section{INTRODUCTION}

Spontaneous ignition is a phenomenon that occurs in a material without external heat supply. In a spontaneous ignition case, heat can be generated, e.g., through a chemical or biological process in the object. When the generated heat can not be adequately dissipated into the surrounding environment, temperature rises and this in turn further speeds up the exothermic oxidation process to eventually result in a self-ignition.

The self-ignition of fuel storages with a possible transition into endogenous fires can cause big economic loss and represents a direct hazard to the storage site and the surrounding environment. Due to the economic and safety concern, it is of great interest to investigate how the relevant parameters and conditions can affect the spontaneous ignition so that people may be able to find out under which conditions the spontaneous ignition can occur and how to avoid it. Thus, a guideline for safe and economic fuel storage can be obtained for practical fuel storing application.

Previously, much experimental and theoretical effort [1-8] has been devoted to this area. In particular, theoretical research has been of a major focus, aiming at development of theoretical tools for prediction of spontaneous ignition. However, in the traditional theoretical analysis, including the well-known classic Frank-Kamenetskii theory [1], simplifications are made in many aspects such as the heat and mass transfer, chemical reaction, geometry and boundary conditions. Unfortunately, these assumptions can be highly invalid in many practical situations. This seriously limits the applicability of the simplified analysis. Recently, people have started to turn to numerical modeling, which has the possibility to solve a set of coupled partial differential equations and thus offers the advantage of high flexibility to simultaneously take into account different coupled processes.

Depending on the devoted effort, numerical modelling can still be of different levels of complexity and capability. In [3], numerical calculation was performed to study coal stockpile's self-ignition in a confined space based on a one-dimensional diffusion model. A two-dimensional calculation with inclusion of convection was carried out in [4] to examine the spontaneous ignition of open coal stockpile. In these two computations, the moisture effect was assumed to be unimportant. Calculation with consideration of moisture was reported in [5,6] to study spontaneous ignition of coal stockpile. A three dimensional diffusion model was presented in $[7,8]$ to study spontaneous ignition in dust accumulation.

This study presents validation of a comprehensive three-dimensional CFD model for simulation of spontaneous ignition of different biomass fuels including sawdust and wood pellets. Experimental measurements were performed to measure temperature history in small scale biomass fuel storages. The performed experiments were then simulated using a parallel finite volume CFD code SMAFS [9]. The simulation was based on solution of a set of unsteady governing equations including the continuity equation, extended Darcy momentum equations, energy conservation equations for both gas and solid phases, and mass conservation equations for different chemical species. With consideration given to a series of essential physical and chemical processes, the drying and transient developing of the temperature field inside the biomass fuel storage were all computed. The computation was performed essentially blindly in a sense that although the experimental results existed prior to computation, no artificial tuning was made in computation to fit experimental results. The numerical results were compared with experimental measurement, showing excellent agreement 


\section{THEORETICAL MODELS}

In the numerical modeling of self-ignition in a biomass fuel storage which is a porous media, a set of coupled governing partial differential equations are solved to obtain the storage's state evolvement. The governing equations, including the continuity equation, momentum equations, mass transport equations for chemical species and energy equations for both gas and solid phases are listed below.

Continuity equation:

$\varphi \frac{\partial \rho_{g}}{\partial t}+\frac{\partial\left(\rho_{g} u_{i}\right)}{\partial x_{i}}=S_{g a s}$

Momentum equation:

$$
\varphi \frac{\partial\left(\rho_{g} u_{i}\right)}{\partial t}+\frac{\partial\left(\rho_{g} u_{i} u_{j}\right)}{\partial x_{j}}=\varphi^{2}\left\{\begin{array}{l}
-\frac{\partial p}{\partial x_{i}}-\frac{\mu}{\kappa} u_{i}+\frac{\partial}{\varphi \partial x_{j}}\left[\mu\left(\frac{\partial u_{i}}{\partial x_{j}}\right)\right] \\
-\frac{C_{F} \rho_{g}}{\kappa^{0.5}}\left(u_{i} u_{i}\right)^{0.5} u_{i}+\rho_{g} a_{g i}
\end{array}\right\}
$$

Mass conservation equation for chemical species:

$$
\varphi \frac{\partial\left(\rho_{g} Y_{i}\right)}{\partial t}+\frac{\partial}{\partial x_{j}}\left(\rho_{g} Y_{i} u_{j}\right)=\varphi \frac{\partial}{\partial x_{j}}\left[\frac{\mu}{S c} \frac{\partial Y_{i}}{\partial x_{j}}\right]+S_{Y_{i}}
$$

Energy conservation equation for gas phase:

$$
\varphi \frac{\partial\left(\rho_{g} H_{g}\right)}{\partial t}+\frac{\partial}{\partial x_{j}}\left(\rho_{g} H_{g} u_{j}\right)=\varphi \frac{\partial}{\partial x_{j}}\left[\frac{\mu}{\operatorname{Pr}} \frac{\partial H_{g}}{\partial x_{j}}\right]+S_{H_{g}}
$$

Energy conservation equation for solid phase:

$$
\frac{\partial\left(\rho_{s} H_{s}\right)}{\partial t}=\frac{\partial}{\partial x_{j}}\left[\frac{\lambda_{s}}{C_{p}} \frac{\partial H_{s}}{\partial x_{j}}\right]+S_{H_{s}}
$$

The moisture balance in solid:

$$
-\frac{\partial\left(\rho_{s} W\right)}{\partial t}=r_{w}
$$


In order to close the above equations, the source terms at the right hand side must be given based on the solved variables.

In the continuity equation, the mass source term can be due to moisture evaporation/condensation and solid phase chemical reaction which consumes oxygen and produces other chemical gases.

In the conservation equations for chemical species, the source term is highly related to the source term in the gas phase continuity equation. For example, for the water vapour, the contribution can be due to condensation/evaporation. For the important oxygen, there is a mass consumption due to chemical reaction.

The source term in the gas phase energy equation is largely due to the convective heat transfer between solid and gas at the solid particle surface. The convective heat transfer between gas and solid particles is very complicated and highly dependent on gas flow velocity and particle size. Due to the high complexity and the relatively low temperature before the spontaneous ignition starts, the radiative heat transfer inside the porous fuel bed is not computed.

In the energy conservation equation for the solid phase, the above discussed convective heat exchange between gas and solid, the moisture condensation/evaporation and the reaction can all contribute to the source term. The energy related to water condensation/evaporation is simply the product of the condensation/evaporation rate and the water's evaporation latent heat. The overall heat production rate due to reaction was provided by experimental measurement. In the simulated experiment which is briefly presented below, temperature history was measured and the data was analyzed to derive the overall reaction rate constant and activation energy.

Another energy source is the energy associated with the mass source appeared in the continuity equation.

Because of the complexity of the problem, the formulation of the above-mentioned source terms is rather complicated. Due to the limited space, the detailed source term formulation is not presented here. Readers are referred to [10] for details.

\section{THE EXPERIMENT}

The spontaneous ignition experiments simulated in this study are basket heating tests. The main equipment used for the experiments is a temperature-controlled oven of $0.34 \mathrm{~m}$ (depth) x $0.40 \mathrm{~m}$ (height) $\times 0.40 \mathrm{~m}$ (width) with re-circulating air. In the experiment, a stainless-steel $0.6 \mathrm{~mm}$ mesh basket filled with solid fuel was suspended in the oven, as shown in Fig. 1. The size of the basket is $0.1 \mathrm{~m} \times 0.1 \mathrm{~m} \times 0.1 \mathrm{~m}$. In order to trigger and speedup the spontaneous ignition process in the experiment, the oven was heated up and maintained to have a temperature of $200^{\circ} \mathrm{C}$ for the sawdust case and $200^{\circ} \mathrm{C}$ for the wood pellets case.

In order to monitor the temperature evolution inside the basket, five $0.25 \mathrm{~mm}$ type $\mathrm{K}$ thermocouples were placed between the volume centre of the basket and a surface center of the basket at one side. The distances between these thermocouples and the volume center are $0 \mathrm{~mm}, 10 \mathrm{~mm}, 20 \mathrm{~mm}, 35 \mathrm{~mm}$ and about $48 \mathrm{~mm}$ (close to the mesh at the side of the basket, with some solid fuel between thermocouple and mesh). 
The biomass fuels used in this study include wood sawdust, and wood pellets of $6 \mathrm{~mm}$ in diameter.

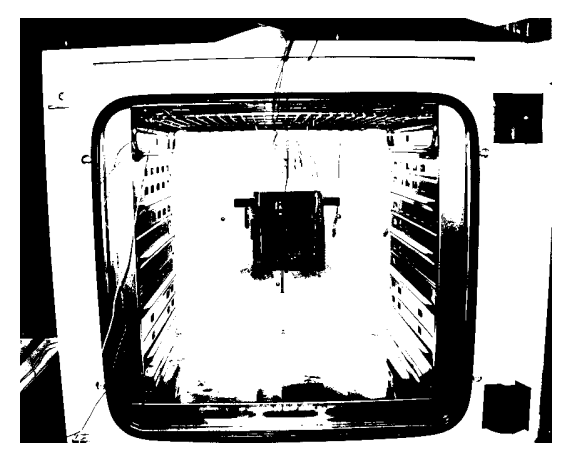

Fig. 1. The experiment setup (inside the oven is the basket filled with biomass fuel).

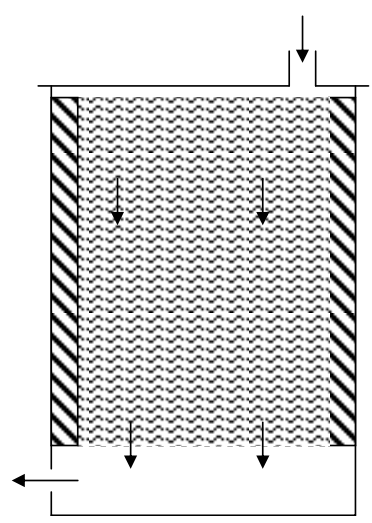

Fig. 2. A sketch of permeability measurement set-up.

\section{THE INPUT DATA ON MATERIAL PROPERTIES}

In order to carry out the CFD simulations, reliable input data on the material's properties is needed. The needed input data covers fuel storage's permeability, porosity, moisture content of the concerned biomass fuel, the compact density of the solid fuel, fuel's overall reaction rate, and the thermal properties of the solid fuel including its specific heat and heat conductivity. Measurements were conducted to provide the necessary material properties. Due to the limited space, it is not feasible to provide here the measurement details. In particular, below we briefly present the permeability measurement which is of importance.

\section{Permeability Measurement Set-up}

The permeability tests were performed in an ad hoc set-up consisting of a standing cylinder of 0.9 meter in height and $0.55 \mathrm{~m}$ in diameter. Figure 2 shows a sketch of the experiment set-up.

The cylinder is closed in the top and the bottom leaving only small holes for air flow. It works as a pressure container and the air volume above the test material will have a constant pressure thus yielding an even vertical flow from top to bottom through the material.

The biomass material is resting on a raft, a stainless steel sheet with distributed circular holes leaving about $50 \%$ of the area free.

Measurements of pressure was taken at a few centimeters above and below the test material respectively with a Furness Micromanometer connected to plastic tubes.

With the measured pressure drop and flow rate, based on Darcy law, the permeability $k$ was calculated as 
$k=\frac{\dot{V}}{A} \frac{h}{\Delta p} \mu$

where $\dot{V}$ is the volume flow rate, $A$ the section area of cylinder and $h$ the height of biomass fuel bed in the cylinder.

\section{List of Input Material Properties}

For completeness, Table 1 gives a list of the input material properties.

Table 1. List of input material properties (all in SI units).

\begin{tabular}{|l|c|c|}
\hline \multicolumn{1}{|c|}{ Material } & Sawdust & 6 mm wood pellets \\
\hline Compact dry density & 400 & 1190 \\
\hline Specific heat & 1700 & 1700 \\
\hline Conductivity & 0.1 & 0.17 \\
\hline Bulk porosity & 0.50 & 0.52 \\
\hline Moisture content & $16 \%$ & $8.2 \%$ \\
\hline Bulk permeability & $1.0 \mathrm{E}-08$ & $6.0 \mathrm{E}-08$ \\
\hline
\end{tabular}

It should be pointed out that the permeability for sawdust case was not directly measured, but calculated using an estimation formula $\frac{D_{p}^{2} \varphi^{3}}{150(1.0-\varphi)^{2}}$ [6].

Due to the space limit, the important fuel's overall reaction rate is not discussed and listed here.

\section{NUMERICAL ASPECTS}

Due to the symmetry of the problem on both width and depth directions (because of the buoyancy, the problem is not symmetric on the vertical direction), a quarter of the sawdust basket was simulated.

A uniform mesh of $20 \times 20 \times 20$ cells was used to provide a fine spatial resolution for the considered quarter of the biomass fuel basket which is of $0.05 \mathrm{~m} \mathrm{x} 0.10 \mathrm{~m} \times 0.05 \mathrm{~m}$. The simulation time step varied from 0.5 to 2 seconds.

The computation was performed using a parallel CFD code SMAFS [9]. Since the temporal state variation is rather smooth and the grid number is small, in this study, the computation was run on a notebook PC of Pentium $41.6 \mathrm{GHz}$ processor with a total CPU time of a few hours.

\section{INITIAL AND BOUNDARY CONDITIONS}

The biomass fuel used in the simulated experiment was conditioned before it was placed into the oven. It was at a temperature of $23^{\circ} \mathrm{C}$ with moisture content of $16 \%$ for sawdust and $8.2 \%$ for $6 \mathrm{~mm}$ wood pellets. The biomass fuel basket was assumed to be homogeneous and isotropic in the simulation. 
When the biomass fuel basket was suspended in the oven, it was subject to both radiative and convective heating from all the six sides.

For the radiative heating, the local net radiation heat flux imposed on biomass fuel basket surface was calculated as $5.67 \times 10^{-8} \times \mathcal{E} \times\left(T_{\text {oven }}^{4}-T_{\text {fuel edge }}^{4}\right)$, where $\mathcal{E}$ is the solid fuel's emissivity.

For the convective heating, since there was a fan operating in the oven, as an approximation, constant convective heat transfer coefficient of $15 \mathrm{~W} / \mathrm{m}^{2} \mathrm{~K}$ was assumed.

For gas phase computation, the boundary condition at the fuel basket relevant boundaries was imposed by considering the oven environment as a free space.

\section{RESULTS AND DISCUSSIONS}

In order to characterize the self-ignition process, it is best to monitor the temperature history at different locations inside the fuel bed. In the experiment, the variation of temperature with time at five different depths inside the biomass fuel basket was measured to record the temperature history.

As mentioned earlier, this study covers biomass fuels of both sawdust and wood pellets. As one may expect, qualitatively similar results are obtained for both fuels. For convenience of results analysis, although the results for both fuels will be presented, we will focus our analysis on the sawdust case. Unless otherwise stated, similar analysis applies to the case of wood pellets.

Figure 3 shows the predicted and measured temperature history for the five locations (points 1 to 5) which are $0 \mathrm{~mm}, 10 \mathrm{~mm}, 20 \mathrm{~mm}, 35 \mathrm{~mm}$ and about $48 \mathrm{~mm}$ away from the basket volume center. As expected, when the sawdust is subject to the external heating, the temperature at the sawdust basket edge (point 5) immediately starts to increase. With the penetration of heat wave into the sample, the inner points' temperature rises. Due to the lag of the heat wave arrival, the temperature increases at the in-depth points is correspondingly delayed.
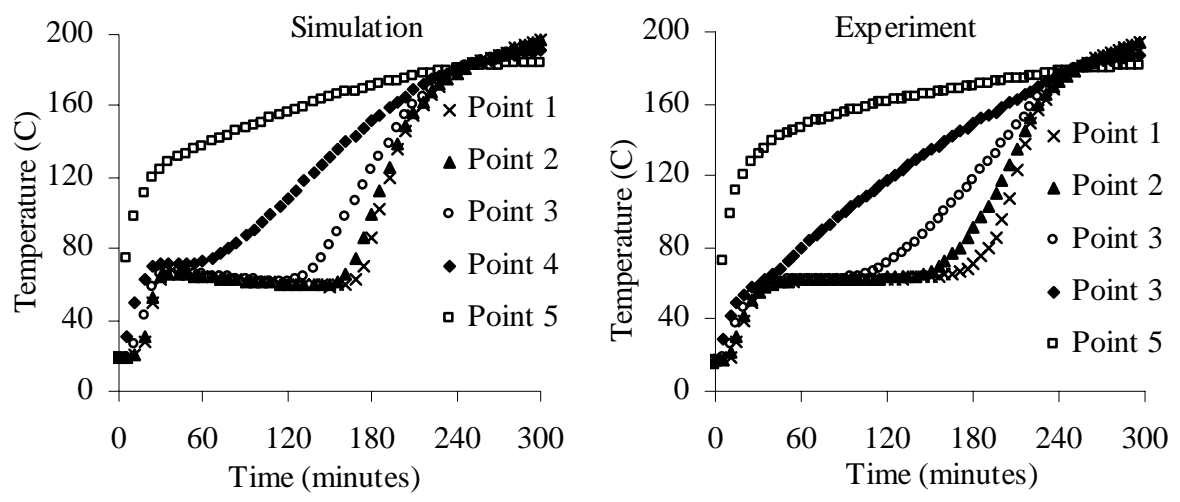

Fig. 3. Predicted and measured temperature history at five locations. 
As can be seen Fig. 3, there is a big difference in temperature rise pattern between the edge point and all the other inner points. At the edge location, the temperature increases steadily with time. However, at all the inner points, the temperature increases and then levels off at about $64^{\circ} \mathrm{C}$. This kind of 'level off' phenomenon starts to appear at the first inner point which is $15 \mathrm{~mm}$ to the fuel storage surface. With increased depth, the 'level off' period becomes longer. After the 'level off' period, the temperature starts to rise again but at a speed which increases with the depth. At about 250 minutes, the temperature curve crosses with each other. This indicates a high potential of spontaneous ignition.

All the above mentioned temperature rise pattern can be explained by examining the solid phase energy equation. For convenience of discussion, we can break the source term into parts which result from different contribution mechanisms and then we can have the expanded solid phase energy equation as following:

$$
\frac{\partial\left(\rho_{s} H_{s}\right)}{\partial t}=\frac{\partial}{\partial x_{j}}\left[\frac{\lambda_{s}}{C_{p}} \frac{\partial H_{s}}{\partial x_{j}}\right]+S_{H_{s}, \text { convection }}+S_{H_{s}, \text { evaporation/condensation }}+S_{H_{s}, \text {,eat production }}
$$

where the source term has been expanded to explicitly denote three components which are respectively convective heat transfer between gas and solid, evaporation/condensation latent heat and heat production by reaction.

In the above expanded solid phase energy equation, the term on the left side denotes the temporal variation of local energy. The different terms on the right hand side of the solid phase energy equation are due to different mechanisms and have different dependences on temperature. The first two terms are due to conduction and convection. If we ignore the dependence of conductivity and convective transfer coefficient on temperature, the heat conduction and convection are linearly proportional to temperature difference. With the increase of local temperature, the heat flux conducted and convected to a local point will decrease and may eventually become negative.

The third term is attributed to evaporation/condensation. It has a rather complicated dependence on temperature. In general, as the wet solid fuel is hit by heat wave, endothermic evaporation happens and the solid fuel starts to dry out. Just behind the drying front, water vapor generated at the drying zone is transported here to meet with cold solid. In this zone, the exothermic condensation may happen.

The last term is the heat production by chemical reaction. This term has an exponential dependence on local temperature. The heat production is of minor importance when the temperature is low at the initial heating stage. However, when the local temperature increases, the local heat production due to chemical reaction increases drastically and then becomes dominant.

With the above analysis of the expanded energy equation, the temperature rise history can be easily explained. As the cold solid fuel is placed in the oven, it is subject to external convective and radiative heating. The outer edge of the fuel storage starts to dry out quickly and temperature steadily increases. Because of the buoyancy resulted from temperature rise, convection is induced. Through diffusion and convection, both the vaporized moisture and heat are transported to the inner parts of fuel storage. Behind the drying front, when the water vapor meets the cold solid fuel, some water vapor condenses 
to release its latent heat. Together with the diffused and convected heat, this released latent heat creates temperature rise in the cold solid. The temperature rise then starts to reduce and eventually stop the condensation and promote evaporation. When the temperature goes up to a certain level, the evaporation can be fast enough to absorb the transported heat. This results in a 'level-off' phenomenon where the drying process is essentially heat transfer controlled. This evaporation also acts as thermal barrier for the heat transfer into deeper locations. Therefore, the 'level-off' period becomes longer in the in-depth points.

After the sawdust has become dried, there is no energy sink to absorb the energy locally produced by chemical reaction and the energy delivered to that local point by conduction and convection. Therefore, the energy is locally stored and local temperature starts to steadily increase. Because of the reaction's exponential dependence on temperature, reaction accelerates quickly with temperature rise and the heat production due to reaction is becoming dominant. At the central part, the produced heat has higher resistance to be dissipated away and the temperature can experience faster rise and may eventually become higher than the temperature at outer part. This is reflected as the curve crossing on the temperature history plot shown in Fig. 3. When the produced heat cannot be sufficiently dissipated away, the reaction may run-away and spontaneous ignition occurs. This is the situation in the present case.

As also can be seen in Fig. 3, the prediction reproduces the experimental measurement very well. The temperature rise pattern, the 'level-off' temperature and temperature crossing time and values are all well predicted. This indicates that all the important processes were well captured by the numerical simulation. To provide a better data comparison, the predicted temperature evolution is compared with measurement for each individual point in Fig. 4.

To better support the above analysis, the predicted temporal variation of moisture content at the above concerned five locations is plotted in Fig. 5. This moisture content history clearly shows the dying process in the biomass fuel storage. As the biomass fuel storage is placed in the hot oven, the fuel edge part dries out quickly. This can be seen from the moisture content history of point 5 . The vaporized moisture is transported into the sample and then meets there with cold solid fuel to condense. Because of the condensation, all the in-depth locations have their moisture contents increased to values higher than the initial moisture content. After reaching their peak moisture contents, the in-depth point starts to dry slowly for a period which becomes longer with increased depth. This slow drying corresponds well with the temperature 'level-off' shown in Fig. 3 and thus support the above analysis. After the slow drying period, followed is a fast drying which matches the temperature's quick increase after its 'level-off' in Fig. 3. To have an overview of spatial distribution of moisture content, a typical visualization of moisture content at a time of 120 minutes is presented in Fig. 6 showing a heart pattern distribution. 

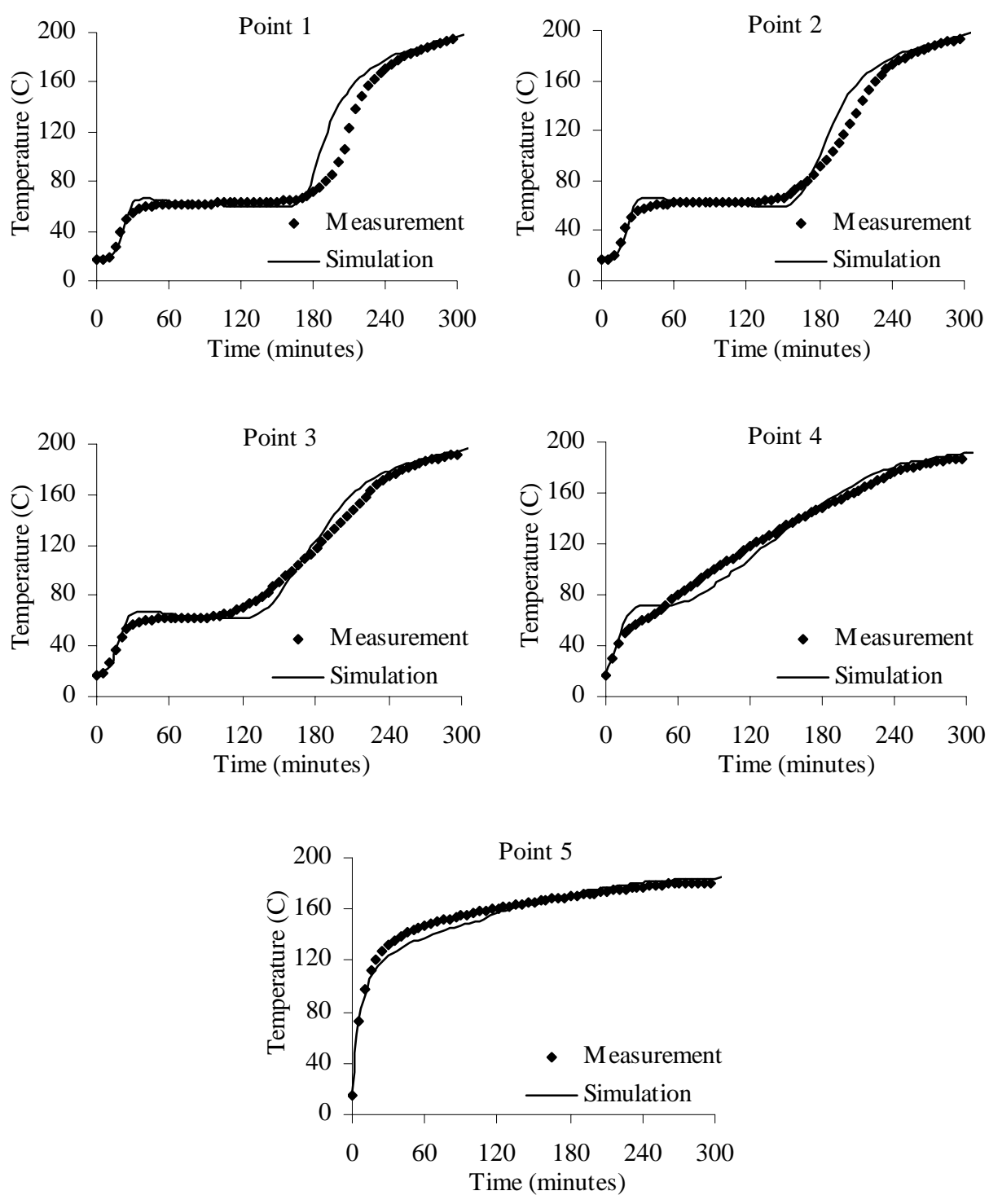

Fig. 4. Point comparison of predicted and measured temperature. 


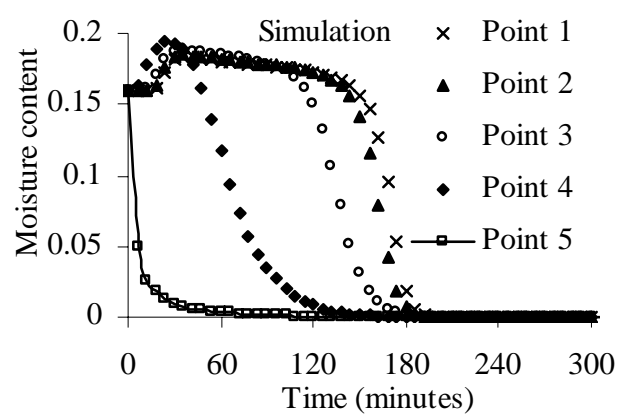

Fig. 5. Predicted time variation of moisture content.

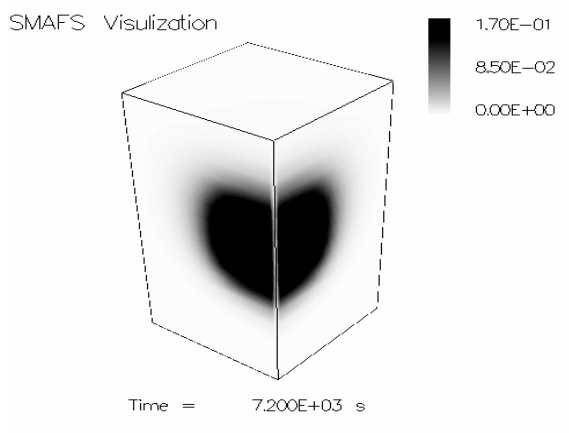

Fig. 6. Spatial distribution of moisture content at $\mathrm{t}=120$ minutes.

As indicated earlier, the solid fuel of $6 \mathrm{~mm}$ wood pellets was also studied. The predicted and measured temperature histories are shown in Figs. 7-8. The results are similar with those for sawdust and good agreement between prediction and measurement is also obtained. More detailed discussion can be found in [10].

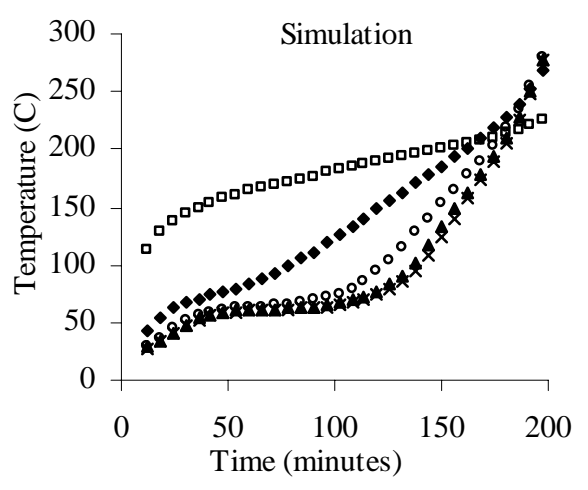

Fig. 7. Predicted temperature for the pellets case (same legend as in Fig. 3).

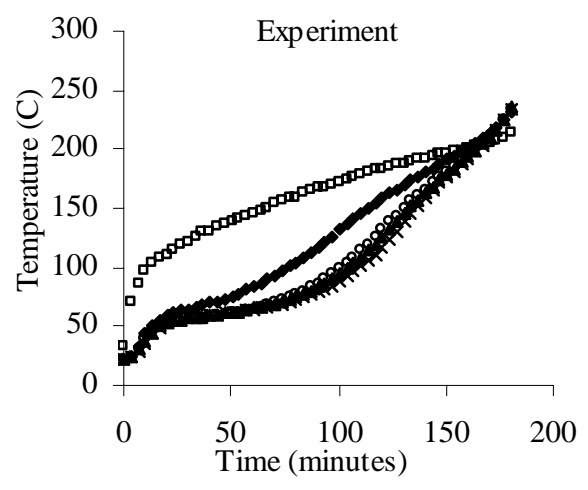

Fig. 8. Measured temperature for the pellets case (same legend as in Fig. 3).

\section{CONCLUSIONS}

Based on numerical solution of a set of governing partial differential equations, comprehensive three dimensional numerical computations were performed to simulate small scale spontaneous ignition experiments. With reliable material properties input data provided by separate measurements, the computations successfully reproduced the measured temperature history at five different locations.

This study reveals the complexity of the concerned spontaneous ignition problem which is due to the strong coupling between flow, heat and mass transfer, water condensation/evaporation and chemical reactions, etc. This complexity prevents possibility of establishing a general and reliable empirical prediction method. The good agreement obtained in this study demonstrates the flexibility and high advantage of CFD 
model in prediction of spontaneous ignition in biomass fuel storage. Future work is planned for both further validations of this CFD simulation technique against large scale experiments and practical industrial applications.

\section{ACKNOWLEDGEMENT}

This work is financed by CECOST, Swedish Fire Protection Association and Swedish Rescue Services Agency which are gratefully acknowledged.

\section{REFERENCES}

[1] Drysdale, D., An Introduction to Fire Dynamics, second edition, Chapter 8, John Wiley and Sons Ltd., New York, 2000.

[2] Akgun, F., and Arisoy, A., "Effect of Particle Size on the Spontaneous Heating of a Coal Stockpile," Combustion And Flame, 99, pp. 137-146, (1994).

[3] Hull, A., Lanthier, J.L., and Agarwal, P.K., "The Role of the Diffusion of Oxygen in the Ignition of a Coal Stockpile in Confined Storage,” Fuel, 76, pp. 975-983, (1997).

[4] Krishnaswamy, S., Agarwal, P.K., and Gunn, R.D., "Low-temperature Oxidation of Coal - Modeling Spontaneous Combustion in Coal Stockpiles," Fuel, 75, pp. 353-362, (1996).

[5] Arisoy, A., and Akgun, F., "Effect of Pile Height on Spontaneous Heating of Coal Stockpiles,” Combust. Sci. and Tech., 153, pp. 157-168, (2000).

[6] Akgun, F., and Essenhigh, R.H., "Self-ignition of Coal Stockpiles: Theoretical Prediction from a Two-dimensional Unsteady-state Model,” Fuel, 80, pp. 409415, (2001).

[7] Krause, U., and Schmidt, M., "The Influence of Initial Conditions on the Propagation of Smouldering Fires in Dust Accumulations," Journal of Loss Prevention in the Process Industries, 14, pp. 527-532, (2001).

[8] Schmidt, M., Lohrer, C., and Krause, U., "Self-ignition of Dust at Reduced Volume Fractions of Ambient Oxygen," Journal of Loss Prevention in the Process Industries, 16, pp. 141-147, (2003).

[9] Yan, Z.H., "SMAFS - A User Guide," private communication, 2004.

[10] Yan, Z.H., and Holmstedt, G., "A Comprehensive CFD Model for Prediction of Spontaneous Ignition in Biomass Fuel Storage,” to be submitted to Combustion And Flame, 2004. 